OPEN ACCESS

Edited by:

Xuejun Wang,

University of South Dakota

United States

Reviewed by:

Md. Shenuarin Bhuiyan Louisiana State University Health Sciences Center, United States

Lie Gao,

University of Nebraska Medical

Center, United States

${ }^{*}$ Correspondence:

Xiyang Peng

xiyangpeng27@aliyun.com

Xiushan Wu

xiushanwu2003@aliyun.com

Ping Zhu

tanganqier@163.com

†These authors have contributed equally to this work

Specialty section: This article was submitted to

Striated Muscle Physiology, a section of the journal

Frontiers in Physiology

Received: 20 July 2020 Accepted: 05 October 2020 Published: 30 November 2020

Citation:

Zhou Z, Zheng L, Tang C, Chen Z, $Z$ Zhu $R$, Peng $X$, Wu $X$ and Zhu $P$ (2020) Identification of Potentially

Relevant Genes for Excessive

Exercise-Induced Pathological Cardiac Hypertrophy in Zebrafish.

Front. Physiol. 11:565307. doi: 10.3389/fphys.2020.565307

\section{Identification of Potentially Relevant Genes for Excessive Exercise-Induced Pathological Cardiac Hypertrophy in Zebrafish}

\author{
Zuoqiong Zhou't, Lan Zheng ${ }^{2 \dagger}$, Changfa Tang ${ }^{2 \dagger}$, Zhanglin Chen ${ }^{2}$, Runkang Zhu', \\ Xiyang Peng ${ }^{2 *}$, Xiushan $W_{u^{1 *}}$ and Ping Zhu ${ }^{1 *}$
}

1 Guangdong Cardiovascular Institute, Guangdong Provincial People's Hospital, Guangdong Academy of Medical Sciences, Guangzhou, China, ${ }^{2}$ Key Laboratory of Physical Fitness and Exercise Rehabilitation of Hunan Province, College of Physical Education, Hunan Normal University, Changsha, China

Exercise-induced cardiac remodeling has aroused public concern for some time, as sudden cardiac death is known to occur in athletes; however, little is known about the underlying mechanism of exercise-induced cardiac injury. In the present study, we established an excessive exercise-induced pathologic cardiac hypertrophy model in zebrafish with increased myocardial fibrosis, myofibril disassembly, mitochondrial degradation, upregulated expression of the pathological hypertrophy marker genes in the heart, contractile impairment, and cardiopulmonary function impairment. Highthroughput RNA-seq analysis revealed that the differentially expressed genes were enriched in the regulation of autophagy, protein folding, and degradation, myofibril development, angiogenesis, metabolic reprogramming, and insulin and FoxO signaling pathways. FOXO proteins may be the core mediator of the regulatory network needed to promote the pathological response. Further, PPI network analysis showed that pik3c3, gapdh, fbox32, fzr1, ubox5, Imo7a, kctd7, fbxo9, lonrf1l, fbx/4, nhpb2/1b, nhp2, fbl, hsp90aa1.1, snrpd3/, dhx15, mrto4, ruvb/1, hspa8b, and faub are the hub genes that correlate with the pathogenesis of pathological cardiac hypertrophy. The underlying regulatory pathways and cardiac pressure-responsive molecules identified in the present study will provide valuable insights for the supervision and clinical treatment of pathological cardiac hypertrophy induced by excessive exercise.

Keywords: pathological cardiac hypertrophy, RNA-seq, autophagy, FoxO signaling pathway, excessive exercise

\section{INTRODUCTION}

The most frequent medical cause of death in athletes is sudden cardiac death (SCD), which usually occurs during intensive training (Harmon et al., 2014). Despite the widely recognized benefits of regular exercise and physical activity, excessive exercise may lead to increased risk of arrhythmia, myocardial ischemia, myocardial fibrosis, and even sudden cardiac death (Siscovick et al., 1984; La Gerche et al., 2012; Galderisi et al., 2015). Given the increasing extensive development of 
mass sports, uncovering the pathogenic mechanism of exerciserelated cardiac disease is urgently required for the prevention of cardiac injury and death.

Moderate exercise regimes can induce benign physiological cardiac hypertrophy, resulting in increased ventricular stroke volume and cardiac output (Pelliccia et al., 1991, 1999). There is strong evidence that exercise is related to a reduced risk of cardiovascular disease, brought about by counteracting structural and functional pathological cardiac changes (Garciarena et al., 2009; Oliveira et al., 2009; Melo et al., 2014; Ooi et al., 2014). However, long-term intense exercise leads to pathological cardiac hypotrophy and heart failure. An increase in cardiomyocyte apoptosis, necrosis, autophagic cell death, and fibrosis occur after long-term excessive training, which could induce the shift from physiological cardiac hypertrophy to pathological cardiac hypotrophy (Carraro and Franceschi, 1997; Nakai et al., 2007; Oka and Komuro, 2008). The focus of research to date has been on cellular and molecular mechanisms of physiological cardiac hypertrophy induced by exercise due to its cardioprotective effects (Ooi et al., 2014). However, the protective and destructive mechanisms induced by exercise are at the root of the development of pathogenic cardiac hypotrophy. In overtraining, an imbalance between cell damage and immune system response leads to pathological remodeling of the heart and, eventually, to heart failure (Schultz et al., 2007). Some of the physiological activities of cardiomyocytes, including cell fibrosis, protein synthesis, mitochondrial dysfunction, metabolic reprogramming, and angiogenesis, have been implicated in pathological hypertrophy, but the specific molecular mechanism and molecular regulatory network of exerciseinduced pathogenic cardiac hypotrophy remains unclear. Understanding this relationship will enable effective prevention and control of exercise overload and heart injury, and improve the medical supervision of athletes.

Zebrafish has become a popular vertebrate model to study the pathogenesis of human diseases, such as cardiomyopathy, because of its convenience and amenability for genetic manipulation, genetic proximity, and relatively small genome (Bournele and Beis, 2016; Dvornikov et al., 2018). To gain understanding of the mechanisms by which excessive exercise exerts adverse cardiac effects, we constructed the first zebrafish overtraining model with the phenotype of pathological cardiac hypotrophy, and applied RNA-seq to systematically identify the key genes and signaling pathways involved in adverse cardiac remodeling in response to excessive exercise.

\section{MATERIALS AND METHODS}

\section{Zebrafish Husbandry and Swimming Training}

$\mathrm{AB}$ strain zebrafish were raised under $14 \mathrm{~h}$ of light at $28^{\circ} \mathrm{C}$ under standard husbandry conditions. Sixty male zebrafish with the same parent, same age, and similar body length and weight were randomly divided into two groups. Before training, the critical swimming velocity of zebrafish (Ucrit) was tested according to a previously reported protocol using Loligo ${ }^{\circledR}$ Systems (\#SW10600)
(Palstra et al., 2010). Zebrafish were subjected to exercise in a current of $24 \mathrm{~cm} / \mathrm{s}$, which corresponds to $80 \%$ of their initial Ucrit, for 4 weeks ( $6 \mathrm{~h}$ for 6 days per week). The control group zebrafish remained in housing tanks for 4 weeks.

\section{Transmission Electron Microscopy}

Zebrafish heart samples were dissected and fixed with 2.5\% glutaraldehyde in $0.1 \mathrm{M} / \mathrm{L}$ phosphate buffer for $12 \mathrm{~h}$. The fixation buffer was discarded and samples were washed with phosphate buffered saline (PBS) for $1 \mathrm{~h}$ and then fixed with $1 \%$ osmium tetroxide at $4^{\circ} \mathrm{C}$ for $2 \mathrm{~h}$. Samples were dehydrated in a series of ethanol concentrations, embedded in Eponate 12 Resin (Ted Pella, United States), and processed into ultrathin sections. The ultrathin sections were stained with uranyl acetate and lead citrate and examined under a Hitachi H7700 transmission electron microscope.

\section{Masson Staining}

Heart samples from each group were harvested, fixed in $4 \%$ paraformaldehyde, embedded in paraffin, and sectioned. The sections were deparaffinized, rehydrated through a series of alcohol concentrations, and washed in distilled water. The sections were then stained in Biebrich scarlet-acid fuchsin solution and washed in distilled water again. The sections were differentiated in phosphomolybdic-phosphotungstic acid solution, stained with aniline blue solution, and rinsed in distilled water. The sections were differentiated in $1 \%$ acetic acid solution, washed in distilled water, dehydrated in a series of ethyl alcohol concentrations, cleared in xylene, and mounted with resinous mounting medium for microscopic analysis.

\section{Measurement of the Rate of Consumption of Oxygen}

Consumption of oxygen was also conducted in Loligo Systems with a mini swim tunnel respirometer, consisting of a fiber optic oxygen probe, DAQ-M automated oxygen measurement system, and AutoRespTM 1 software. Measurement of the rate of consumption of oxygen $\left(\mathrm{MO}_{2}\right)$ was performed by automated intermittent-flow respirometry in loops of $7 \mathrm{~min}$. Each loop consisted of a $5 \mathrm{~min}$ measuring phase followed by a $90 \mathrm{~s}$ flushing phase and $30 \mathrm{~s}$ waiting phase. Before the measurement, the zebrafish fasted for $24 \mathrm{~h}$ and swam at a speed of $0.8 \mathrm{bl} / \mathrm{s}$ for $2 \mathrm{~h}$ to eliminate the sense of stress. After this acclimation period, the velocity was steadily increased ( $2.7 \mathrm{bl} / \mathrm{s}$ every $20 \mathrm{~min}$ ) until the fish were unable to continue swimming due to fatigue. Concentrations of oxygen $\left(\mathrm{O}_{2}\right)$ were measured using a fiber optic oxygen dipping probe which was connected to a Fibox 3 mini-sensor oxygen meter (Precision Sensing $\mathrm{GmbH}$, Regensburg, DE).

\section{Reverse Transcriptase Quantitative PCR (RT-qPCR)}

Total RNA was isolated from tissue using TRIzol (Invitrogen, CA) according to the manufacturer's protocol. To analyze transcriptional changes, cDNA was generated using Superscript III reverse transcriptase (Invitrogen, CA), and RT-qPCR was 
performed using SYBR Green PCR Master Mix (Takara, Dalian, China). Standard PCR conditions were used to measure transcript abundance for 35 cycles in an ABI7500 machine (ABI Biosystems, Columbia, MD). The delta-delta Ct method was used to calculate the relative abundance of the tested genes. Primers used for expression analysis are listed in Supplementary Table 1.

\section{Identification of Differentially Expressed Genes}

RNA was extracted from the hearts of the training group and the control group zebrafish to construct a cDNA library, which was sequenced in paired mode on an Illumina HiSeq6000. Clean data were obtained from the raw data after discarding adapter sequences and low-quality sequences using Trimmomatic (Bolger et al., 2014). We used the DESeq2 package to identify the differentially expressed genes (DEGs) between the two groups. DEGs were selected when the $P$-value was less than 0.05 .

\section{Gene Ontology (GO) and Kyoto Encyclopedia of Genes and Genomes (KEGG) Enrichment Analysis of DEGs}

Gene enrichment analysis was performed on the DEGs using the DAVID online database to identify physiological changes in overloaded hearts. A $P<0.05$ was considered to be statistically significant for enrichment. KEGG pathway analysis was then used to identify biochemical pathways that were enriched for the DEGs, with an adjusted $p$-value of less than 0.05 .

\section{Clusters of Orthologous Groups (COGs) Analysis of DEGs}

We used the bioMart package to obtain the protein sequences of the DEGs, which were used for COGs analysis on the EggNOG online tool ${ }^{1}$.

\section{Protein-Protein Interaction (PPI) Network Construction}

The PPI network was predicted using the search tool for the retrieval of interacting genes $\left(\right.$ STRING) ${ }^{2}$ online database. Interactions with a combined score of $>0.4$ were considered to be statistically significant. The bioinformatics platform Cytoscape ${ }^{3}$ was used to visualize the molecular interaction network.

\section{Statistical Analysis}

Data were analyzed using $\mathrm{R}$ software, version 3.6.3 ( $\mathrm{R}$ Core Team, 2020), to estimate the homogeneity of variance, and Student's $t$-tests were performed to compare the variance between experimental groups. A $p<0.05$ was considered to be statistically significant.

\section{Echocardiography}

Zebrafish in each group were anaesthetized in $0.16 \mathrm{mg} / \mathrm{mL}$ tricaine solution for $2 \mathrm{~min}$ and placed with the ventral

\footnotetext{
${ }^{1}$ http://eggnog5.embl.de/\#/app/home

${ }^{2}$ https://string-db.org/

${ }^{3}$ https://cytoscape.org
}

side up in a small sponge holder. Echocardiography was then performed using a VisualSonics Vevo 2100 System echocardiograph (VisualSonics, Toronto, CA, United States) as described previously (Fang et al., 2020).

\section{RESULTS}

\section{Excessive Exercise Leads to Pathologic Cardiac Hypertrophy}

After 4 weeks of excessive exercise, zebrafish hearts were dissected for microscopic observation. The hearts of zebrafish that were subjected to excessive exercise were evidently enlarged compared to those of the control zebrafish (Figures 1A,B and Supplementary Figure S1). Masson's trichrome staining was performed to detect the accumulation of collagen fibrosis in the heart. According to the observed bright blue staining, collagen fibrosis was accumulating in both the interstitial and perivascular regions of the excessively exercised group (Figures 1E,F), while trichrome staining in the control zebrafish hearts was marginal (Figures 1C,D). The percentage of myocardial fibrosis area in the excessively exercised group was significantly larger than the control group (Figure 1G). The ultrastructure of the heart was analyzed using a transmission electron microscope. The muscle fibers and mitochondria were neatly arranged in the cardiomyocytes of the control group, while the muscle fibers and mitochondria of the excessively exercised group were arranged in a disorderly manner, and mitochondrial structural abnormalities and autophagy were observed (Figures 1H,I). Further detection of mitochondrial functional markers showed that mitochondrial cytochrome C protein Cyc1, NADH dehydrogenase 1 (nd1), $\mathrm{NADH}$ dehydrogenase 2 (nd2), NADH dehydrogenase 5 (nd5), and NADH dehydrogenase 6 (nd6) were significantly downregulated (Figure 1J). Echocardiographic analysis showed that zebrafish with excessive exercise-induced pathologic cardiac hypotrophy developed significant contractile impairment compared to the control group (Figure 1K and Supplementary Movies 1, 2). Oxygen consumption was used as an indicator of cardiopulmonary function. The maximal oxygen consumption of zebrafish after excessive exercise decreased significantly compared to in the control group (Figure 1L). RT-qPCR analysis showed that the embryonic genes $n p p a, n p p b$, and $m y h 7$ showed elevated expression levels, while the members of the classic physiological signaling pathway pik3r1/PI3K, mapk3/ERK1, eif4a/eif4ea, and map2k2a/MEK2 were downregulated in the hearts of excessively exercised zebrafish compared to in the control group (Figure 1M). Taken together, these changes in the cardiac tissue suggest that excessive exercise can lead to pathologic cardiac hypertrophy.

\section{Differential Expression of Genes Caused by Excessive Exercise}

High-throughput transcriptome analysis was used to unravel the underlying mechanisms of pathologic cardiac hypertrophy. We found that 1,519 genes $(5.82 \%)$ were specifically expressed 

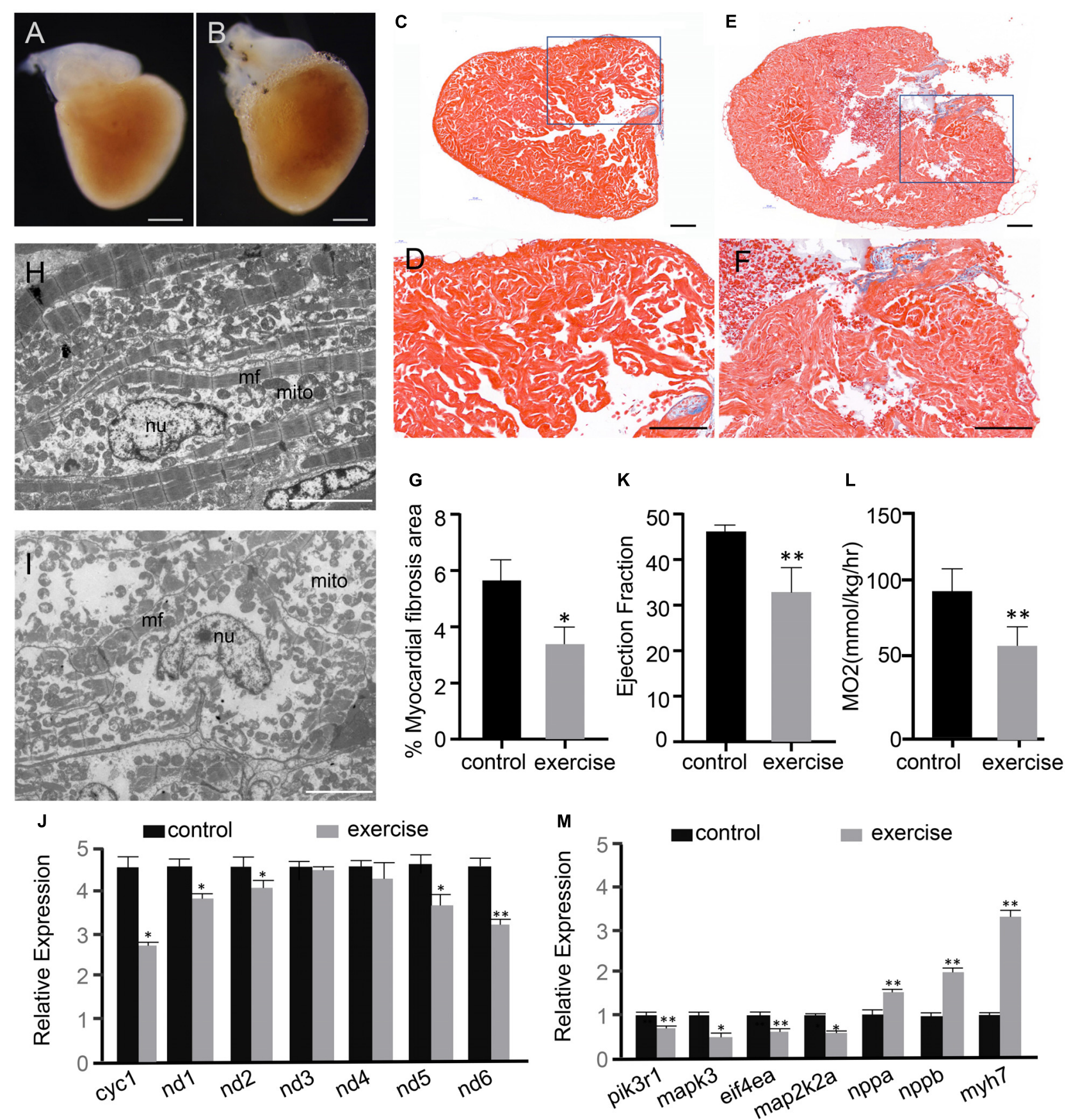

FIGURE 1 | Excessive exercise leads to pathological cardiac hypertrophy. (A,B) Representative images from control (A) and excessively-exercised zebrafish hearts (B) after 4 weeks of static and excessive-exercise treatment. (C-F) Representative pictures of Masson's trichrome-stained ventricular tissues of control (C,D) and excessively-exercised zebrafish hearts (E,F). Blue area indicates fibrosis. (D,F) Are enlargements of the rectangle area in (C,E), respectively. Scale bar $=100 \mu \mathrm{m}$. (G) Quantitative analysis of Masson staining positive myocardial fibrosis area using Image J. ${ }^{*} p<0.05$ by unpaired Student's $t$-test. $n=3$. (H,I) TEM images of control (H) and excessively exercised heart tissue (I). Scale bar $=5 \mu \mathrm{m}$. (J) RT-qPCR analysis of the expression of mitochondrial functional markers. ${ }^{*} p<0.05$, ${ }^{* *} p<0.01$ by unpaired Student's $t$-test. (K) Echocardiographic analysis of zebrafish after excessive exercise and in the static control (LVPW, LV posterior wall thickness). Error bars indicate SEM. $n=4$. (L) The maximal oxygen consumption $\left(\mathrm{MO}_{2}\right)$ of zebrafish after excessive exercise and in the control group was analyzed. $n=8$. (M) RT-qPCR analysis of the expression of pathologic and physiological hypotrophy-related marker genes. ${ }^{*} p<0.05,{ }^{* *} p<0.01$ by unpaired Student's $t$-test.

and 1,212 genes $(4.64 \%)$ were absent in zebrafish hearts with pathological hypotrophy (Figure 2A) compared to in the control group. A total of 3,082 mRNAs were differentially expressed with a $p<0.05$ in paired control and pathologic hypertrophy heart samples, of which 1,569 mRNAs were upregulated and 1,513 were downregulated (Figure 2B). 
A

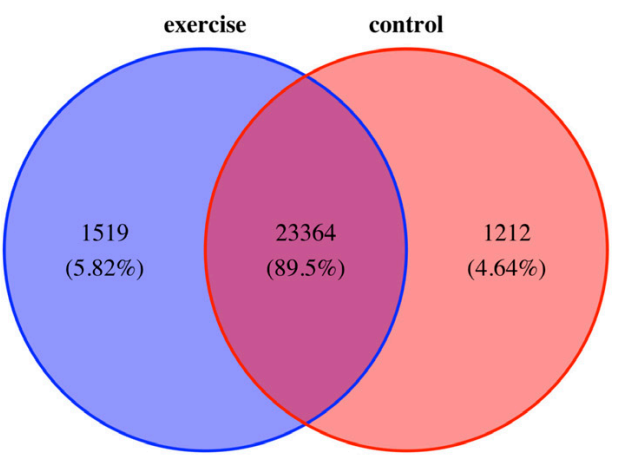

B

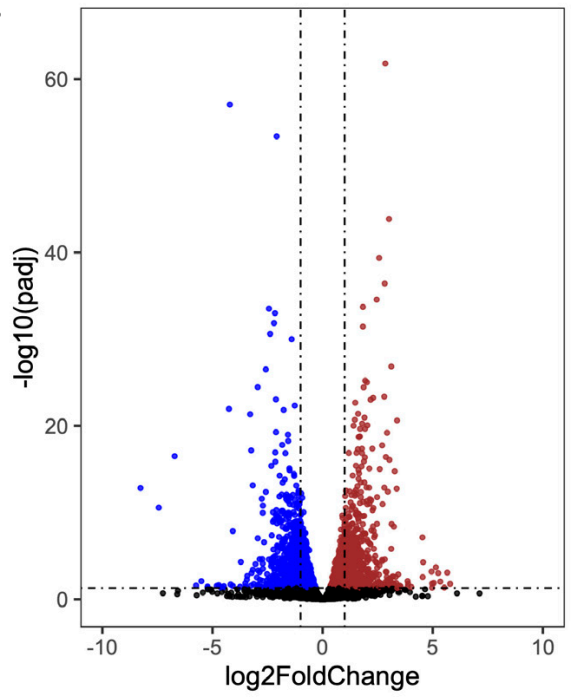

- UP

- DOWN

- NOT

C

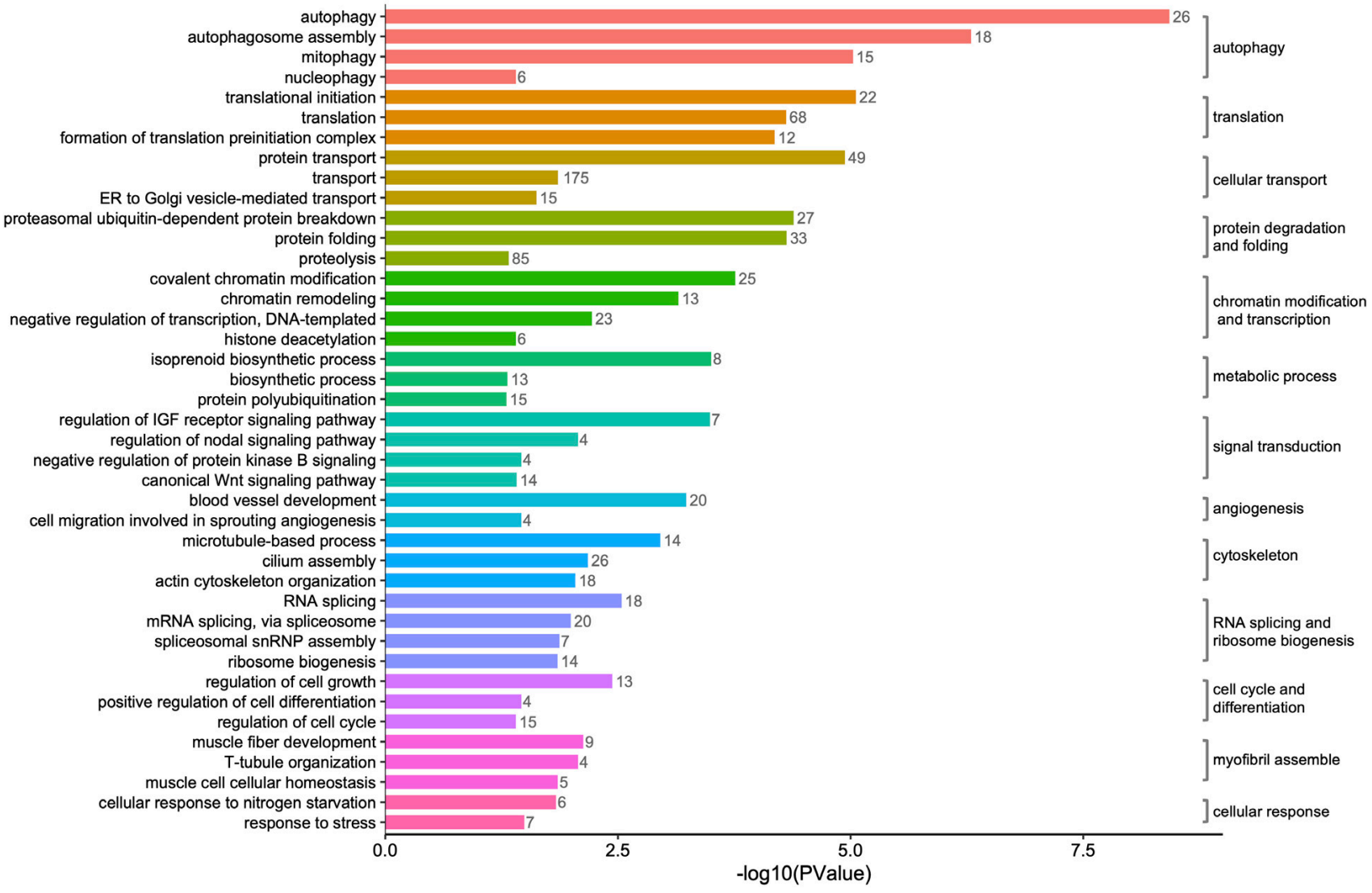

FIGURE 2 | Identification of differentially expressed genes (DEGs) and enrichment analysis of GO terms in zebrafish hearts after excessive exercise and in the control group. (A) Venn diagram of the total number of identified genes in the excessive exercise and control groups. (B) Volcano plot constructed using fold-change values and $P$ adj-values. Red: upregulated, Blue: downregulated. (C) The DEG enrichment analysis of GO terms in the biological process subcategory. The number indicates the number of DEGs that are enriched in the clustered representative terms. 


\section{Functional Annotation of DEGs}

Gene ontology enrichment analysis was performed to investigate the biological functions of all DEGs. Autophagy, autophagosome assembly, mitophagy, translational initiation, protein transport, regulation of translational initiation, proteasomal ubiquitindependent protein breakdown, protein folding, translation, formation of translation preinitiation complex, and covalent chromatin modification were the top 10 enriched GO terms in the "biological processes" subcategory (Supplementary Table 2). Under the "molecular function" subcategory, the significantly enriched terms $(p<0.05)$ were associated with autophagy, transcription, cellular transport, protein folding and degradation, chromatin modification, and transcription, signal transduction, angiogenesis, cytoskeleton, RNA splicing, and ribosome biogenesis, cell cycle, and differentiation, myofibril assembly, metabolic process, and cellular response (Figure 2C).

To improve the specificity of the GO enrichment analysis, this process was repeated for genes that were up- or downregulated by at least twofold in the hearts of zebrafish exposed to excessive exercise. The upregulated genes were enriched in pathways related to autophagosome assembly, mitophagy, protein ubiquitination, and lipid, and fructose metabolic process. The downregulated genes were significantly enriched in response to heat, protein folding, sarcomere organization, regulation of insulin like growth factor receptor signaling pathway, and myofibril assembly (Figures $\mathbf{3 A}, \mathbf{B}$ ). We classified the DEGs $(p<0.05$, fold change $>2)$ using the COG database, and identified that signal transduction mechanisms, posttranslational

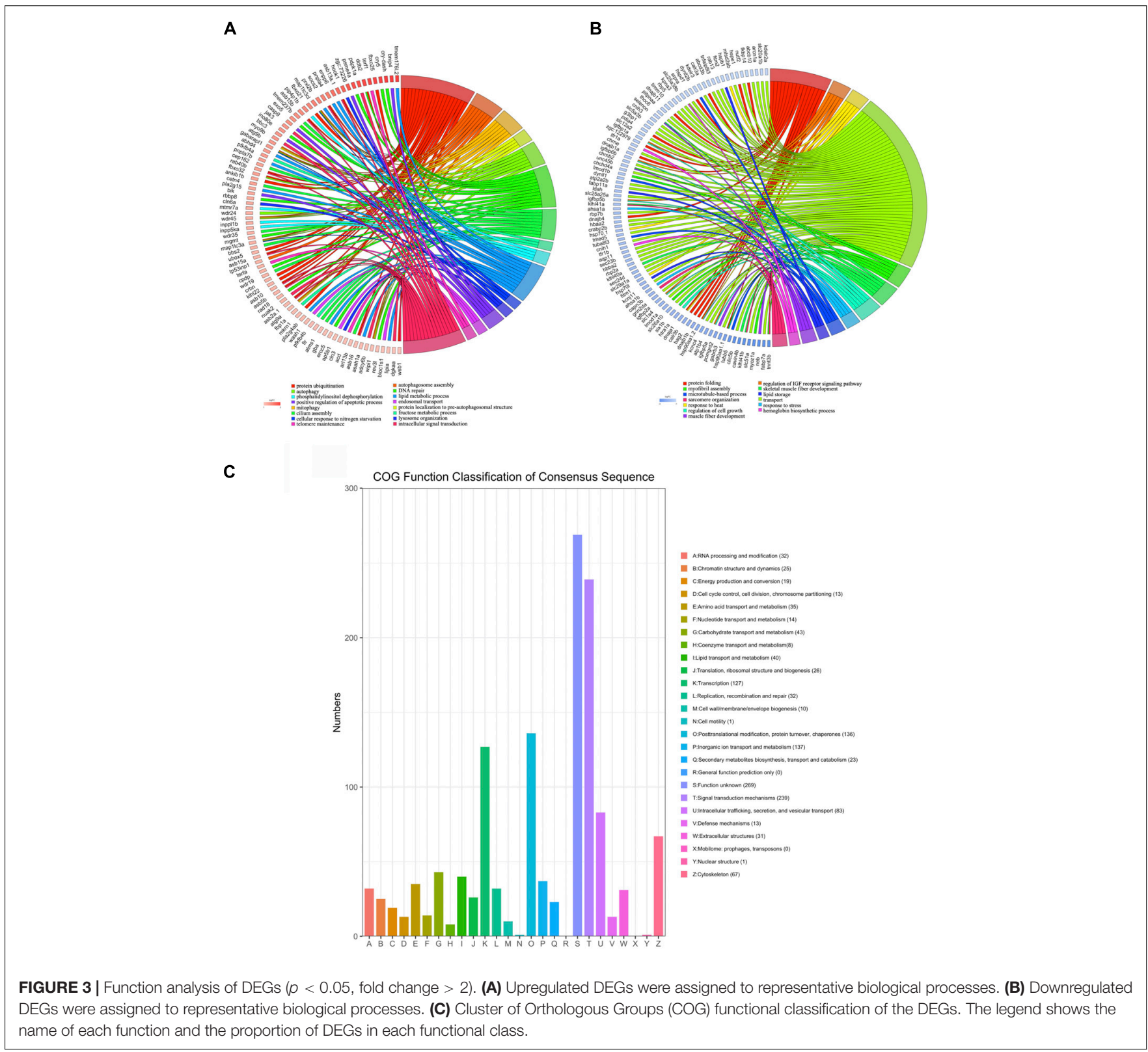


modification, protein turnover, chaperons, and transcription were the most representative functional clusters (Figure 3C).

Next, we aimed to identify the high-level GO terms that lead to pathological hypotrophy. We validated the expression of genes enriched in these GO terms and found that the expression of genes involved in autophagy (atg101, agt9a, atg9b, tsc1, and tsc2), FoxO signaling pathway (foxo1, foxo4, and fbox32), insulin/insulin-like signaling (insra, igf1rb, irs, and irs $2 a)$, glycolysis ( $f b p 1 a$ and $p f k f b 4 a)$, and angiogenesis inhibitor hif3a were up-regulated (Figure 4A). Genes involved in protein folding ( $h$ sp90aa1.1, hsp90aa1.2, hsp70.1, and hspad8b), myofibril assembly (cavin4b, dag1, tcap3, and tnnt3), angiogenesis (vegfc, $p d g f b r$, and hifla), and fatty acid storage (fitm 1 and fitm2) were down-regulated (Figure 4B).

\section{KEGG Pathway Analysis of DEGs}

We mapped the DEGs to biological pathways using the KEGG pathway database. Most of the upregulated genes were enriched in lysosome, insulin resistance, regulation of autophagy, insulin signaling pathway, FoxO signaling pathway, mTOR signaling pathway, other glycan degradation, nucleotide excision repair, fructose, and mannose metabolism. Most of the downregulated genes were enriched in protein processing in the endoplasmic reticulum, spliceosome, proteasome, terpenoid backbone biosynthesis, focal adhesion, protein export, RNA transport, ribosome biogenesis in eukaryotes, phagosome, and tight junction (Table 1 and Supplementary Table 3).

\section{Construction of PPI Network and Cluster Identification}

A PPI network was generated using the STRING database. The genes pik3c3, gapdh, fbxo32, fzr1, ubox5, lmo7a, kctd7, fbxo9, lonrfll, and $f b x l 4$ were the top ten upregulated genes with the highest fold change (Table 2), and $n h p 2 l 1 b$, nhp2, fbl, hsp90aa1.1, snrpd3l, dhx15, mrto4, ruvbl1, hspa8b, and faub were the top 10 downregulated genes with the highest fold change (Table 2). The PPI network of these hubgenes with the interaction of DEGs was generated using Cytoscape (Figures 5A,B). We performed an additional round of GO enrichment analysis of the proteins in the PPI network. The representative 10 BP GO terms are listed in Table 3. We found that the most downregulated genes were involved in protein synthesis and modification, mitochondrial function, and sarcomere structure, while the most upregulated genes were involved in autophagy, signal transduction, and glycolytic process.

\section{DISCUSSION}

Cardiac hypertrophy initially takes place as an adaptive response to hemodynamic overload to reduce ventricular wall stress. Whether it results in pathological or physiological hypertrophy depends on different underlying molecular mechanisms (Nakamura and Sadoshima, 2018). Due to the convenience of manipulation and the relatively simple genome, we have designed several swimming schemes with different durations, frequencies, and swimming speeds to train zebrafish model to study human cardiac pathological and physiological hypertrophy. Physiological cardiac hypertrophy is characterized by normal or enhanced cardiac function and normal organization of cardiac structure, whereas pathological cardiac hypertrophy is accompanied by fibrosis and cardiac dysfunction (McMullen and Jennings, 2007). In the present study, we over-trained zebrafish according to a pre-designed swimming program and found that the hearts of these zebrafish were enlarged, with increased myocardial fibrosis

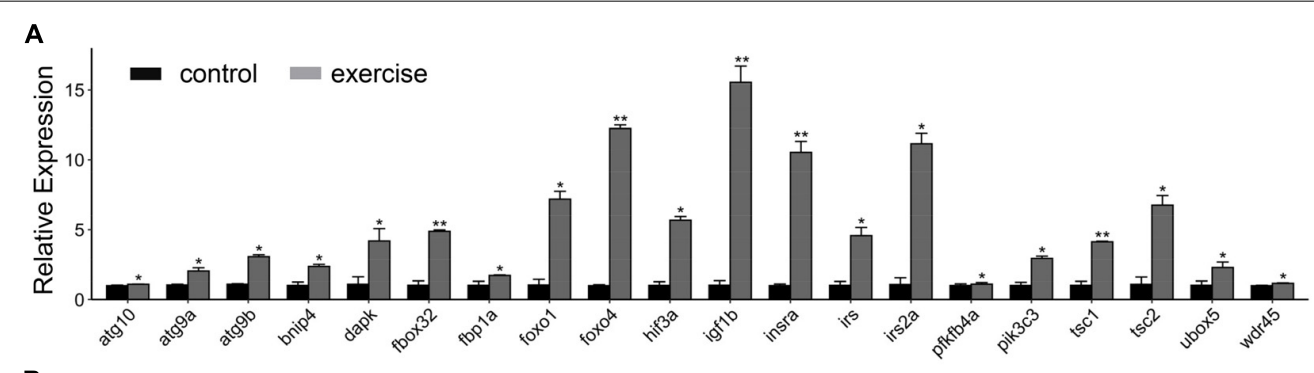

B

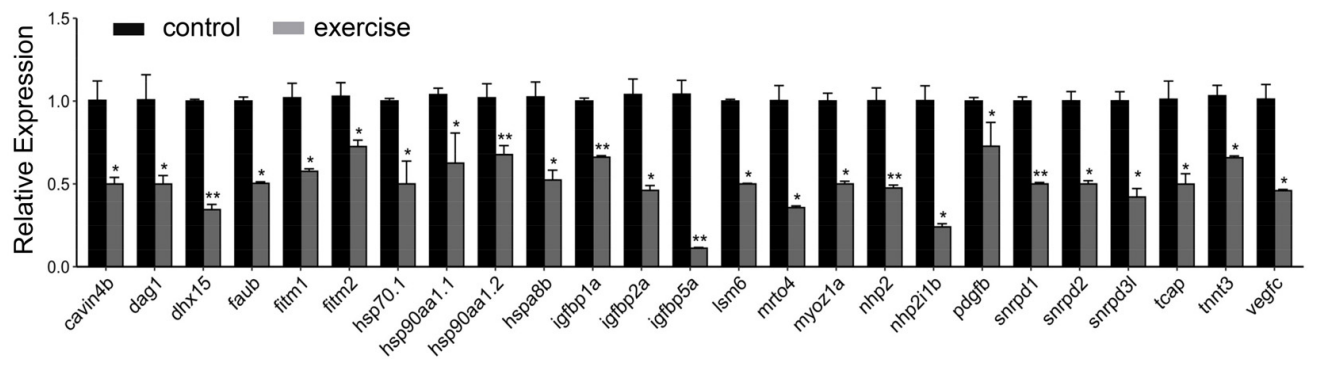

FIGURE 4 | RT-qPCR validation of key genes involved in excessive exercise induced pathological cardiac hypertrophy. (A) upregulated genes involved in pathological remodeling. (B) downregulated genes involved in pathological remodeling. $n=8$ in control and exercise group. ${ }^{*} p<0.05$, ${ }^{\star \star} p<0.01$ by unpaired Student's $t$-test. 
TABLE 1 | Enriched KEGG pathways in the heart tissue of excessively exercised zebrafish.

\begin{tabular}{lllcc}
\hline \multicolumn{2}{c}{ ID } & Term & Count & P-value \\
\hline Up & dre04142 & Lysosome & 26 & $3.27 \mathrm{E}-06$ \\
& dre04931 & Insulin resistance & 20 & $6.97 \mathrm{E}-04$ \\
& dre04140 & Regulation of autophagy & 8 & $9.27 \mathrm{E}-04$ \\
dre04910 & Insulin signaling pathway & 21 & $6.23 \mathrm{E}-03$ \\
dre04068 & FoxO signaling pathway & 20 & $1.14 \mathrm{E}-02$ \\
dre04150 & mTOR signaling pathway & 11 & $1.52 \mathrm{E}-02$ \\
dre00511 & Other glycan degradation & 6 & $1.60 \mathrm{E}-02$ \\
dre03420 & Nucleotide excision repair & 8 & $2.01 \mathrm{E}-02$ \\
dre00051 & Fructose and mannose metabolism & 7 & $4.38 \mathrm{E}-02$ \\
Down & dre04141 & Protein processing in endoplasmic & 51 & $6.99 \mathrm{E}-12$ \\
& reticulum & & \\
dre03040 & Spliceosome & 33 & $1.53 \mathrm{E}-06$ \\
dre03050 & Proteasome & 18 & $1.44 \mathrm{E}-05$ \\
dre00900 & Terpenoid backbone biosynthesis & 10 & $1.42 \mathrm{E}-04$ \\
dre04510: & Focal adhesion & 44 & $1.89 \mathrm{E}-04$ \\
dre03060: & Protein export & 8 & $4.32 \mathrm{E}-03$ \\
dre03013 & RNA transport & 27 & $6.52 \mathrm{E}-03$ \\
dre03008 & Ribosome biogenesis in eukaryotes & 15 & $1.53 \mathrm{E}-02$ \\
dre04145 & Phagosome & 24 & $3.18 \mathrm{E}-02$ \\
dre04530 & Tight junction & 18 & $4.19 \mathrm{E}-02$ \\
& & & \\
\hline
\end{tabular}

TABLE 2 | Hub genes in the PPI networks.

\begin{tabular}{|c|c|c|c|}
\hline & Hub gene & Description & Node \\
\hline \multirow[t]{10}{*}{ Up } & pik3c3 & Phosphatidylinositol 3-kinase, catalytic subunit type 3 & 52 \\
\hline & gapdh & Glyceraldehyde-3-phosphate dehydrogenase & 49 \\
\hline & $\mathrm{fbxo32}$ & F-box protein 32 & 45 \\
\hline & fzr1 & Fizzy/cell division cycle 20 related $1 b$ & 45 \\
\hline & ubox5 & U-box domain containing 5 & 44 \\
\hline & Imo7a & LIM domain only $7 a$ & 44 \\
\hline & kctd7 & BTB/POZ domain-containing protein & 44 \\
\hline & fbxo9 & F-box only protein 9 & 43 \\
\hline & lonrf1l & $\begin{array}{l}\text { LON peptidase } \mathrm{N} \text {-terminal domain and ring finger } 1 \text {, } \\
\text { like }\end{array}$ & 43 \\
\hline & $\mathrm{fbx} / 4$ & F-box and leucine-rich repeat protein 4 & 42 \\
\hline \multirow[t]{10}{*}{ Down } & nhp211b & NHP2 non-histone chromosome protein 2-like 1b & 105 \\
\hline & nhp2 & NHP2 ribonucleoprotein homolog & 104 \\
\hline & $\mathrm{fbl}$ & Fibrillarin & 101 \\
\hline & hsp90aa1.1 & Heat shock protein HSP 90-alpha 1 & 99 \\
\hline & snrpd3l & Small nuclear ribonucleoprotein D3 polypeptide, like & 97 \\
\hline & dhx15 & DEAH (Asp-Glu-Ala-His) box polypeptide 15 & 97 \\
\hline & mrto4 & MRT4 homolog, ribosome maturation factor & 96 \\
\hline & ruvbl1 & RuvB-like AAA ATPase 1 & 96 \\
\hline & hsap8b & Heat shock $70 \mathrm{kDa}$ protein 8 & 96 \\
\hline & faub & FAU ubiquitin like and ribosomal protein S30 fusion b & 96 \\
\hline
\end{tabular}

and disassembled myofibril and mitochondria. The hearts of zebrafish subjected to over-exercise had notable contractile impairment and cardiopulmonary function impairment, suggesting the occurrence of pathological cardiac hypertrophy. We carried out high-throughput sequencing analysis of the transcripts in pathologically hypertrophied zebrafish hearts and the hearts of a matched control group. The DEGs between the two groups were mainly involved in autophagy, protein homeostasis, myofibril assembly, angiogenesis, metabolic reprogramming, the insulin/insulin-like growth factor (IGF) signaling pathway, and the FoxO signaling pathway. These signaling pathways and factors related to these physiological changes may form a regulatory network of exercise-induced pathological hypertrophy (Figure 6).

\section{Insulin and the IGF Signaling Pathway}

The insulin and IGF signaling pathways regulate a series of cellular processes in the heart, including cell metabolism, contractility, growth, proliferation, differentiation, and apoptosis (Saltiel and Kahn, 2001). The binding of insulin or IGF1 to the insulin receptor (insr) or IGF1R recruits and phosphorylates the adapter proteins insulin receptor substrate 1 (IRS1) and IRS2, which in turn activates the PI3K-AKT signaling pathway to preserve physiological cardiomyocyte growth (Heineke and Molkentin, 2006; Duan et al., 2010).

Although the Insulin/PI3K/AKT axis is known to promote heart function under stress by inducing physiological hypertrophy of the heart, the insulin signals may also induce pathological hypertrophy (Shimizu et al., 2010). Excessive cardiac insulin signaling or constitutive activation of the PI3K/AKT pathway could disrupt the coordination between tissue growth and angiogenesis in the heart, and cause cardiac dysfunction. In mice, PI3K/AKT upregulation after chronic pressure overload induces pathological hypertrophy of the heart (Shioi et al., 2002). Increasing clinical evidence has shown that insulin resistance, as a result of hyperinsulinemia, is the main cause of non-ischemic heart failure (Witteles and Fowler, 2008). Likewise, our KEGG analysis of DEGs in excessively exercised zebrafish hearts showed that the insulin pathway and insulin resistance were significantly enriched, and the observed unregulated expression of insr(insra), $i g f 1 r b$, irs(irs1, irs2b,irs2a), pi3k(pik3cb, pik3cg), pdk(pdpk1b), and akt(akt2) may facilitate the activation of cardiac insulin signals. Interestingly, the FoxO proteins (foxola and foxo4) were upregulated, which may inhibit some key functions of insulin or insulin-like growth factor, including cell metabolism, autophagy, protein synthesis, and myofibril assembly (Lee and Dong, 2017; Link, 2019). These two signaling pathways may thus contribute to pathological cardiac hypotrophy.

In contrast, some of the zebrafish insulin-like growth factor binding proteins (igfbp), such as igfbpla, igfbp2a, igfbp5a, igfbp5b, and igfbp6b, were significantly downregulated in the exercise group. The IGFBP family is a highly conserved secreted protein, and is an important regulator of the IGF signaling pathway (Firth and Baxter, 2002). These proteins mediate the signal transmission of IGFs, mainly by regulating the half-life and access of IGF-1 to its receptors on the cell membrane surface (Eivers et al., 2004; Holly and Perks, 2006). Increased IGF1 levels in serum are associated with physiological cardiac hypotrophy in athletes (Neri Serneri et al., 2001). IGFBP2 plays an essential role in maintaining physiological hypertrophy (Olszanecka et al., 2017). The increased synthesis of IGFBP2 may be related to a protective mechanism against excessive cardiac remodeling (Rehfeldt et al., 2010; Sharples et al., 2013). Low levels of IGFBP2 are independently associated with a 
A
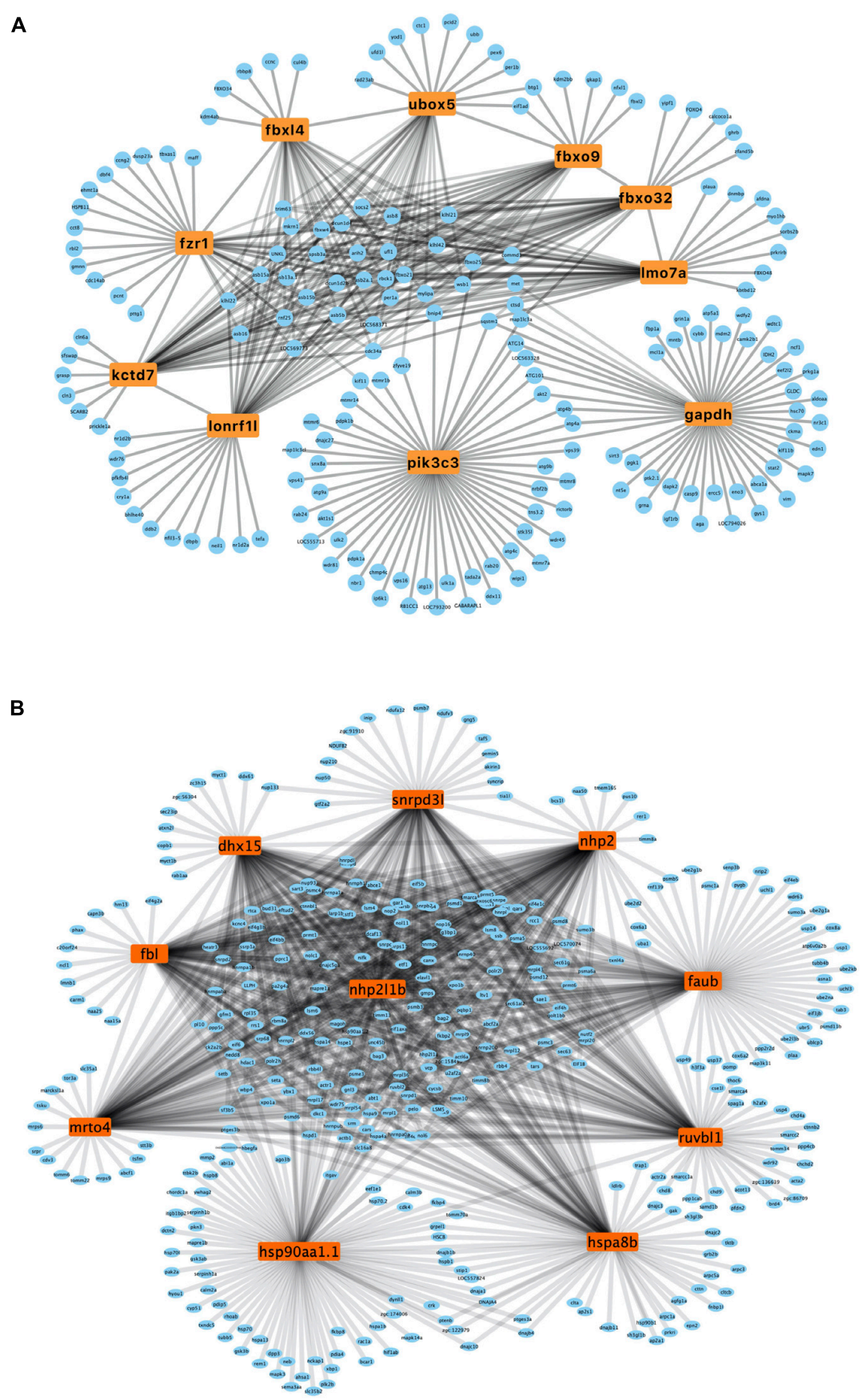

FIGURE 5 | PPI network of upregulated and downregulated genes. (A) PPI network of upregulated hub genes. Hub genes are listed in the box. (B) PPI network of downregulated hub genes. Hub genes are listed in the box.

lower stroke volume index, which is a powerful predictor of poor prognosis in patients with aortic stenosis (Carter et al., 2015). Low stroke volume in patients with aortic stenosis is a typical symptom of left ventricular concentric remodeling, which initially compensates for increased cardiac pressure load, but then leads to fibrosis and heart failure. In the present study, significant downregulation of igfbp $2 a$, igfbp $1 a$, igfbp $5 a$, igfbp5b, and igfbp $6 b$ was detected, which may be one of the possible reasons for cardiac fibrosis and heart failure in excessive exercise-induced pathological hypotrophy.

In general, insulin and IGF signals are associated with physiological cardiomyocyte growth. In this excessive exerciseinduced pathological hypotrophy model, the activation of cardiac insulin and IGF signaling is also accompanied by the upregulation of the FoxO signaling pathway and may be a cause of cardiac pathological remodeling. 
TABLE 3 | Gene ontology enrichment analysis for the intersecting proteins of hub genes in the heart tissue of zebrafish subjected to excessive exercise.

\begin{tabular}{|c|c|c|c|}
\hline & ID & Term & $P$-value \\
\hline \multirow[t]{10}{*}{ Up } & GO:0000045 & Autophagosome assembly & $2.49 \mathrm{E}-14$ \\
\hline & GO:0016567 & Protein ubiquitination & 4.34E-14 \\
\hline & GO:0000422 & Mitophagy & $9.89 \mathrm{E}-12$ \\
\hline & GO:0044804 & Nucleophagy & $1.23 \mathrm{E}-05$ \\
\hline & GO:0035556 & Intracellular signal transduction & $5.32 \mathrm{E}-05$ \\
\hline & GO:0051697 & Protein delipidation & $9.85 \mathrm{E}-04$ \\
\hline & GO:0016311 & Dephosphorylation & $1.02 \mathrm{E}-03$ \\
\hline & GO:0006096 & Glycolytic process & $5.16 \mathrm{E}-03$ \\
\hline & GO:0006995 & Cellular response to nitrogen starvation & $6.21 E-03$ \\
\hline & GO:0006289 & Nucleotide-excision repair & $1.86 \mathrm{E}-02$ \\
\hline \multirow[t]{11}{*}{ Down } & GO:0006457 & Protein folding & $4.50 \mathrm{E}-14$ \\
\hline & GO:0006412 & Translation & $8.92 \mathrm{E}-10$ \\
\hline & GO:0006511 & $\begin{array}{l}\text { Ubiquitin-dependent protein catabolic } \\
\text { process }\end{array}$ & $3.25 \mathrm{E}-05$ \\
\hline & GO:0006396 & RNA processing & $3.15 \mathrm{E}-05$ \\
\hline & GO:0042254 & Ribosome biogenesis & $3.41 E-04$ \\
\hline & GO:0006950 & Response to stress & 3.57E-04 \\
\hline & GO:0006123 & $\begin{array}{l}\text { Mitochondrial electron transport, } \\
\text { cytochrome c to oxygen }\end{array}$ & $1.96 \mathrm{E}-03$ \\
\hline & GO:0006338 & Chromatin remodeling & 2.27E-02 \\
\hline & GO:0030833 & Regulation of actin filament polymerization & 3.06E-02 \\
\hline & GO:0007017 & Microtubule-based process & $3.51 E-02$ \\
\hline & GO:0006886 & Intracellular protein transport & 7.67E-04 \\
\hline
\end{tabular}

\section{Protein Homeostasis}

We identified several key protein synthesis pathways associated with DEGs in the hearts of excessively exercised zebrafish, suggesting a reduction in the capacity to produce functional proteins. The expression of spliceosome components, such as snrpd1, snrpd2, and 1 sm6, was downregulated, which has the effect of decreasing the initiation of synthesis of proteins in cardiomyocytes. We also identified the downregulation of proteins involved in protein endoplasmic reticulum processing, especially hsp70s (hsp70.1/hspa8b) and hsp90s (hsp90aa1.1/2), and their co-chaperones (Figure 3), which may lead to aberrant protein folding (Hartl et al., 2011). The accumulation of misfolded proteins can cause endoplasmic reticulum pressure, promote the expression of dapk protein, and induce protein ubiquitin degradation and autophagy. Correspondingly, the proteins involved in autophagosome assembly, mitophagy, autophagy, and protein localization to pre-autophagosomal structures were upregulated after excessive exercise. The upregulation of autophagy induction complex-tcs $1 /$ tcs2 and "core" autophagy machinery, such as the ulk1 complex (ulk1a, ulk2, atg13, atg101, and rb1cc1), pik3c3 complex (atg14 and pik3c3), atg8 conjugation system (map1l3c3a, map1l3c3cl, atg4c, and gabarapb), and atg9 complex (atg9a, atg9b, wipil, and wdr45) may provide a protective mechanism for protein folding disorders to maintain proteostasis (Mathai et al., 2017). Increased mitophagy is an indicator of mitochondrial damage and dysfunction, and is one of the typical features of pathological hypotrophy (Nakamura and Sadoshima, 2018).

\section{Altered Sarcomere Structure and the Blockade of Myofibril Development}

The expression of the effectors of the FoxO pathway, such as foxo $1 a$, foxo4, fbox 32 , and fbox 25 , was upregulated in the hearts of excessively exercised zebrafish, which may lead to myopathy (Shimizu et al., 2017). Pathological cardiac hypertrophy is the most common primary cardiomyopathy, which represents a disease with decreased myocardial contractility and insufficient cardiac pump function. The cause of many cardiomyopathies is the structural abnormality of the cardiac myofibrils (Brieler et al., 2017; Dadson et al., 2017; Lin et al., 2019).

According to enrichment analysis, myofibril development and sarcomere organization were affected in zebrafish hypertrophic hearts. The downregulation of related genes, such as tnnt $3 b$, myozla, klhl41a/b, hsp90aa1.1, and unc45b, influences the assembly, repair, and contraction of myofibrils. Mutants for klhl41 and klhl40 have disrupted muscle structure and loss of movement (Gupta et al., 2013; Ravenscroft et al., 2013). Likewise, unc45b and hap90aa1.1 play an essential role in the assembly of thick filaments of sarcomeres, and the functional

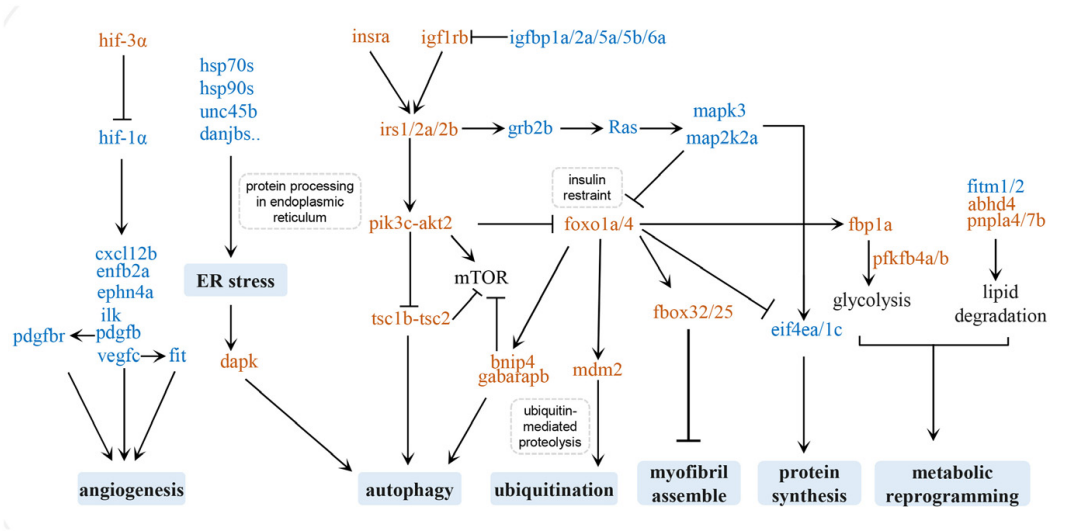

FIGURE 6 | Regulatory network of exercise-induced pathological hypertrophy. Upregulated genes are in brown and downregulated genes are in blue. 
deletion of unc $45 \mathrm{~b}$ resulted in a complete loss of motility and disorder of myofibrils in zebrafish hearts (Etard et al., 2007; Hawkins et al., 2008; Myhre et al., 2014). To restore the homeostasis of myofibril after damage or under stress, hsp90aa1.1 and unc45b dissociate from the $\mathrm{Z}$ line and translocate to the A bands of the myofibril to repair myofibrillar damage and restore sarcomere assembly (Etard et al., 2008). Troponin is the constituent protein of sarcomeres and is essential for the regulation of $\mathrm{Ca}^{2+}$-dependent contraction. In humans, a variety of cardiomyopathies are associated with mutations in all three cardiac troponin subunits (TNNT1, TNNT2, and TNNT3). The depletion of zebrafish tnnt3b, the ortholog of human TNNT3, causes deficiency of troponin activity and sarcomere disintegration, probably due to deregulation of actin-myosin activity (Ferrante et al., 2011).

In addition to the genes affecting sarcomere organization, T-tubule organization-related genes, such as tcap, dag1, dysf, and cavin $4 b$, were also downregulated. The sarcolemma penetrates into the intracellular space of myofilaments to form cardiac T-tubules, which are rich in concentrating voltage-gate L-type calcium channels and signal transduction molecules, and plays a central role in myofibril electrophysiology and sarcomere contraction. Zebrafish dag1, tcap, or cavin $4 b$ mutants display T-tubule abnormalities and progressive muscle dysfunction (Zhang et al., 2009; Gupta et al., 2011; Housley et al., 2016). The dysf zebrafish morphants also showed misaligned and fragmented T-tubules. Ordered proteinmembrane scaffold assembly for sarcolemma repair begins with the formation of the dysf and anxa6 complex at the lesion (Roostalu and Strahle, 2012).

Adult zebrafish cardiomyocytes possess the ability to regenerate, which permits them to restore the heart after substantial amounts of damage, while the inhibition of myofibril development and sarcomere assembly may block the regeneration process. Excessive exercise causes the downregulation of genes that enable regeneration, and therefore, the injured heart is unable to recover to homeostasis, eventually resulting in apoptosis or necrosis.

\section{Insufficient Angiogenesis}

In response to an increase in pump pressure, the myocytes and capillary microvasculature grow harmoniously to increase heart weight, resulting in enlargement (Oka et al., 2014). However, the disproportional growth of myocytes and capillaries leads to insufficient capillary density and coronary flow for oxygen and nutrient supply, resulting in myocardial ischemia in pathologically hypertrophied hearts (Nakamura and Sadoshima, 2018).

Hypoxia-inducible factors (HIFs), which are important transcription factors in response to hypoxia, play an important regulatory role in the angiogenesis of pressure-overloaded hearts. HIFs are heterodimers composed of an unstable $\alpha$-subunit (HIF- $1 \alpha$, HIF- $2 \alpha$, and HIF- $3 \alpha$ ) and a stable $\beta$-subunit (HIF $\beta$ ) (Sousa Fialho et al., 2019). The downregulation of HIF1 $\alpha$ expression or the inhibition of HIF1 $\alpha$ function can reduce angiogenesis and cause maladaptive hypertrophy during chronic pressure overload. We found that the expression of hif $1 \alpha$ (hiflab) was downregulated in excessively exercised zebrafish hearts, while the upregulation of hif $3 \alpha$ might competitively combine with hif $\beta$ (hiflal), which further weakens the function of hifl $\alpha$ (Zhang et al., 2014). At the same time, angiogenesis factors related to hif $1 \alpha$, including pdgfb, vegfc, $\operatorname{cxcl} 12 b$, efnb2a, ephn4a, ilk, and fit1 (Semenza, 2014), were also significantly downregulated in the exercised zebrafish, suggesting that angiogenesis was inhibited in our excessive exercise-induced hypertrophic model.

\section{Metabolic Reprogramming}

Although appropriate metabolic adaptation to pathological hypertrophy may produce harmful intermediate metabolites, cardiomyocytes still undergo metabolic reprogramming to maintain contractile function.

Fructose, mannose, and other glycogen degradation increased during excessive exercise, and the glycolysis rate-limiting enzymes, such as fbpla and $\mathrm{pfkfb} 4 \mathrm{a} / \mathrm{b}$, were significantly upregulated, suggesting that carbohydrate metabolism becomes essential under these conditions (Strohecker et al., 2015). Another significant metabolic change was related to lipid metabolism. The observed downregulation of fitm $1 / 2$ results in a decrease in the storage of triglycerides, the most important source of stored energy in cells, in lipid droplets (Gross et al., 2010). Concurrently the enzymes that decompose triglycerides and phospholipids into fatty acids, such as lipia, gba, pnpla7b, pnpla4, abhd4, prxl2, pla2g15, and asah1a (Schaloske and Dennis, 2006; Gao et al., 2009; Lelieveld et al., 2019), were upregulated, suggesting that the lipids were broken down into fatty acids to provide energy during excessive exercise in zebrafish.

We also observed the downregulation of enzymes related to terpenoid backbone biosynthesis, such as pass1, pdss2, idil, acat2, and ggps1. Terpenoid backbone biosynthesis involves the use of acetyl-CoA to synthesize physiologically active substances (Mendoza-Poudereux et al., 2015). As a metabolite of glycolysis and fatty acid oxidation, acetyl-CoA is mainly used to enter the tricarboxylic acid (TCA) cycle to produce ATP, perhaps owing to the inhibition of terpenoid backbone biosynthesis under the pressure of energy consumption. Interestingly, ubiquinone is the product of terpenoid backbone biosynthesis and is also an important component of the mitochondrial respiratory chain. Thus, disruption of terpenoid backbone biosynthesis may possibly contribute to mitochondrial dysfunction and pathological cardiac remodeling.

\section{DATA AVAILABILITY STATEMENT}

The datasets generated for this study can be found in the NCBI Sequence Read Archie (SRA) accession PRJNA635689.

\section{ETHICS STATEMENT}

The animal study was reviewed and approved by the Ethics Committee of Hunan Normal University (approval number: 2018/046). 


\section{AUTHOR CONTRIBUTIONS}

$\mathrm{XP}, \mathrm{PZ}$, and XW designed the experiments. ZC and RZ collected the zebrafish sample. ZZ, XP, and LZ performed the experiments. $\mathrm{ZZ}, \mathrm{XP}, \mathrm{LZ}$, and CT performed the analysis of the experimental results and RNA-seq. ZZ, XP, XW, and PZ wrote the manuscript. All authors have read and approved the final manuscript.

\section{FUNDING}

This research was funded by the National Key Research and Development Program of China (2018YFA0108700 and 2017YFA0105602), NSFC Projects of International Cooperation and Exchanges (81720108004), National Natural Science Foundation of China $(32071175,81801392$, 81570279,

\section{REFERENCES}

Bolger, A. M., Lohse, M., and Usadel, B. (2014). Trimmomatic: a flexible trimmer for Illumina sequence data. Bioinformatics 30, 2114-2120. doi: 10 . 1093/bioinformatics/btu170

Bournele, D., and Beis, D. (2016). Zebrafish models of cardiovascular disease. Heart Fail. Rev. 21, 803-813. doi: 10.1007/s10741-016-9579-y

Brieler, J., Breeden, M. A., and Tucker, J. (2017). Cardiomyopathy: an overview. Am. Fam. Physician. 96, 640-646.

Carraro, U., and Franceschi, C. (1997). Apoptosis of skeletal and cardiac muscles and physical exercise. Aging 9, 19-34. doi: 10.1007/bf03340125

Carter, S., Capoulade, R., Arsenault, M., Bedard, E., Dumesnil, J. G., Mathieu, P., et al. (2015). Relationship between insulin-like growth factor binding protein2 and left ventricular stroke volume in patients with aortic stenosis. Can. J. Cardiol. 31, 1447-1454. doi: 10.1016/j.cjca.2015.04.024

Dadson, K., Hauck, L., and Billia, F. (2017). Molecular mechanisms in cardiomyopathy. Clin. Sci. 131, 1375-1392. doi: 10.1042/CS20160170

Duan, C., Ren, H., and Gao, S. (2010). Insulin-like growth factors (IGFs), IGF receptors, and IGF-binding proteins: roles in skeletal muscle growth and differentiation. Gen. Comp. Endocrinol. 167, 344-351. doi: 10.1016/j.ygcen. 2010.04.009

Dvornikov, A. V., de Tombe, P. P., and Xu, X. (2018). Phenotyping cardiomyopathy in adult zebrafish. Prog. Biophys. Mol. Biol. 138, 116-125. doi: 10.1016/j.pbiomolbio.2018.05.013

Eivers, E., McCarthy, K., Glynn, C., Nolan, C. M., and Byrnes, L. (2004). Insulin-like growth factor (IGF) signalling is required for early dorso-anterior development of the zebrafish embryo. Int. J. Dev. Biol. 48, 1131-1140. doi: 10.1387/ijdb.041913ee

Etard, C., Behra, M., Fischer, N., Hutcheson, D., Geisler, R., and Strahle, U. (2007). The UCS factor Steif/Unc-45b interacts with the heat shock protein Hsp90a during myofibrillogenesis. Dev. Biol. 308, 133-143. doi: 10.1016/j.ydbio.2007. 05.014

Etard, C., Roostalu, U., and Strahle, U. (2008). Shuttling of the chaperones Unc45b and Hsp90a between the A band and the Z line of the myofibril. J. Cell Biol. 180, 1163-1175. doi: 10.1083/jcb.200709128

Fang, Y., Sun, Y., Luo, C., Gu, J., Shi, Z., Lu, G., et al. (2020). Evaluation of cardiac dysfunction in adult zebrafish using high frequency echocardiography. Life Sci. 253:117732. doi: 10.1016/j.lfs.2020.117732

Ferrante, M. I., Kiff, R. M., Goulding, D. A., and Stemple, D. L. (2011). Troponin $\mathrm{T}$ is essential for sarcomere assembly in zebrafish skeletal muscle. J. Cell Sci. 124(Pt 4), 565-577. doi: 10.1242/jcs.071274

Firth, S. M., and Baxter, R. C. (2002). Cellular actions of the insulin-like growth factor binding proteins. Endocr. Rev. 23, 824-854. doi: 10.1210/er.20010033

Galderisi, M., Cardim, N., D’Andrea, A., Bruder, O., Cosyns, B., Davin, L., et al. (2015). The multi-modality cardiac imaging approach to the Athlete's heart: an
81974019, and 81670290), the Research Team Project of Natural Science Foundation of Guangdong Province of China (2017A030312007), The Key Program of the Guangzhou Science Research Plan (201904020047), the Special Project of the Dengfeng Program of Guangdong Provincial Peoplešs Hospital (DFJH201812, KJ012019119, and KJ012019423), Hunan Provincial Natural Science Foundation of China (No. 2020JJ5354), and Postgraduate Scientific Research Innovation Project of Hunan Province (CX20200532).

\section{SUPPLEMENTARY MATERIAL}

The Supplementary Material for this article can be found online at: https://www.frontiersin.org/articles/10.3389/fphys. 2020.565307/full\#supplementary-material

expert consensus of the European Association of Cardiovascular Imaging. Eur. Heart J. Cardiovasc. Imaging 16:353. doi: 10.1093/ehjci/jeu323

Gao, J. G., Shih, A., Gruber, R., Schmuth, M., and Simon, M. (2009). GS2 as a retinol transacylase and as a catalytic dyad independent regulator of retinylester accretion. Mol. Genet. Metab. 96, 253-260. doi: 10.1016/j.ymgme.2008.12.007

Garciarena, C. D., Pinilla, O. A., Nolly, M. B., Laguens, R. P., Escudero, E. M., Cingolani, H. E., et al. (2009). Endurance training in the spontaneously hypertensive rat: conversion of pathological into physiological cardiac hypertrophy. Hypertension 53, 708-714. doi: 10.1161/HYPERTENSIONAHA. 108.126805

Gross, D. A., Snapp, E. L., and Silver, D. L. (2010). Structural insights into triglyceride storage mediated by fat storage-inducing transmembrane (FIT) protein 2. PLoS One 5:e10796. doi: 10.1371/journal.pone.0010796

Gupta, V., Kawahara, G., Gundry, S. R., Chen, A. T., Lencer, W. I., Zhou, Y., et al. (2011). The zebrafish dag1 mutant: a novel genetic model for dystroglycanopathies. Hum. Mol. Genet. 20, 1712-1725. doi: 10.1093/hmg/ ddr047

Gupta, V. A., Ravenscroft, G., Shaheen, R., Todd, E. J., Swanson, L. C., Shiina, M., et al. (2013). Identification of KLHL41 Mutations Implicates BTB-Kelchmediated ubiquitination as an alternate pathway to myofibrillar disruption in nemaline myopathy. Am. J. Hum. Genet. 93, 1108-1117. doi: 10.1016/j.ajhg. 2013.10.020

Harmon, K. G., Drezner, J. A., Wilson, M. G., and Sharma, S. (2014). Incidence of sudden cardiac death in athletes: a state-of-the-art review. Heart 100, 12271234. doi: 10.1136/heartjnl-2014-093872.rep

Hartl, F. U., Bracher, A., and Hayer-Hartl, M. (2011). Molecular chaperones in protein folding and proteostasis. Nature 475, 324-332. doi: 10.1038/ nature 10317

Hawkins, T. A., Haramis, A. P., Etard, C., Prodromou, C., Vaughan, C. K., Ashworth, R., et al. (2008). The ATPase-dependent chaperoning activity of Hsp90a regulates thick filament formation and integration during skeletal muscle myofibrillogenesis. Development 135, 1147-1156. doi: 10.1242/dev. 018150

Heineke, J., and Molkentin, J. D. (2006). Regulation of cardiac hypertrophy by intracellular signalling pathways. Nat. Rev. Mol. Cell Biol. 7, 589-600. doi: $10.1038 / \mathrm{nrm} 1983$

Holly, J., and Perks, C. (2006). The role of insulin-like growth factor binding proteins. Neuroendocrinology 83, 154-160. doi: 10.1159/000095523

Housley, M. P., Njaine, B., Ricciardi, F., Stone, O. A., Holper, S., Kruger, M., et al. (2016). Cavin4b/Murcb is required for skeletal muscle development and function in zebrafish. PLoS Genet. 12:e1006099. doi: 10.1371/journal.pgen. 1006099

La Gerche, A., Burns, A. T., Mooney, D. J., Inder, W. J., Taylor, A. J., Bogaert, J., et al. (2012). Exercise-induced right ventricular dysfunction and structural remodelling in endurance athletes. Eur. Heart J. 33, 998-1006. doi: 10.1093/ eurheartj/ehr397 
Lee, S., and Dong, H. H. (2017). FoxO integration of insulin signaling with glucose and lipid metabolism. J. Endocrinol. 233, R67-R79. doi: 10.1530/JOE-17-0002

Lelieveld, L. T., Mirzaian, M., Kuo, C. L., Artola, M., Ferraz, M. J., Peter, R. E. A., et al. (2019). Role of beta-glucosidase 2 in aberrant glycosphingolipid metabolism: model of glucocerebrosidase deficiency in zebrafish. J. Lipid Res. 60, 1851-1867. doi: 10.1194/jlr.RA119000154

Lin, Y. H., Yap, J., Ramachandra, C. J. A., and Hausenloy, D. J. (2019). New insights provided by myofibril mechanics in inherited cardiomyopathies. Cond. Med. 2, 213-224.

Link, W. (2019). Introduction to FOXO biology. Methods Mol. Biol. 1890, 1-9. doi: 10.1007/978-1-4939-8900-3_1

Mathai, B. J., Meijer, A. H., and Simonsen, A. (2017). Studying autophagy in zebrafish. Cells 6:21. doi: 10.3390/cells6030021

McMullen, J. R., and Jennings, G. L. (2007). Differences between pathological and physiological cardiac hypertrophy: novel therapeutic strategies to treat heart failure. Clin. Exp. Pharmacol. Physiol. 34, 255-262. doi: 10.1111/j.1440-1681. 2007.04585.x

Melo, S. F., Fernandes, T., Barauna, V. G., Matos, K. C., Santos, A. A., Tucci, P. J., et al. (2014). Expression of MicroRNA-29 and collagen in cardiac muscle after swimming training in myocardial-infarcted rats. Cell Physiol. Biochem. 33, 657-669. doi: 10.1159/000358642

Mendoza-Poudereux, I., Kutzner, E., Huber, C., Segura, J., Eisenreich, W., and Arrillaga, I. (2015). Metabolic cross-talk between pathways of terpenoid backbone biosynthesis in spike lavender. Plant Physiol. Biochem. 95, 113-120. doi: 10.1016/j.plaphy.2015.07.029

Myhre, J. L., Hills, J. A., Jean, F., and Pilgrim, D. B. (2014). Unc45b is essential for early myofibrillogenesis and costamere formation in zebrafish. Dev. Biol. 390, 26-40. doi: 10.1016/j.ydbio.2014.02.022

Nakai, A., Yamaguchi, O., Takeda, T., Higuchi, Y., Hikoso, S., Taniike, M., et al. (2007). The role of autophagy in cardiomyocytes in the basal state and in response to hemodynamic stress. Nat. Med. 13, 619-624. doi: 10.1038/nm1574

Nakamura, M., and Sadoshima, J. (2018). Mechanisms of physiological and pathological cardiac hypertrophy. Nat. Rev. Cardiol. 15, 387-407. doi: 10.1038/ s41569-018-0007-y

Neri Serneri, G. G., Boddi, M., Modesti, P. A., Cecioni, I., Coppo, M., Padeletti, L., et al. (2001). Increased cardiac sympathetic activity and insulin-like growth factor-I formation are associated with physiological hypertrophy in athletes. Circ. Res. 89, 977-982. doi: 10.1161/hh2301.100982

Oka, T., Akazawa, H., Naito, A. T., and Komuro, I. (2014). Angiogenesis and cardiac hypertrophy: maintenance of cardiac function and causative roles in heart failure. Circ. Res. 114, 565-571. doi: 10.1161/CIRCRESAHA.114.300507

Oka, T., and Komuro, I. (2008). Molecular mechanisms underlying the transition of cardiac hypertrophy to heart failure. Circ. J. 72(Suppl. A), A13-A16. doi: 10.1253/circj.cj-08-0481

Oliveira, R. S., Ferreira, J. C., Gomes, E. R., Paixao, N. A., Rolim, N. P., Medeiros, A., et al. (2009). Cardiac anti-remodelling effect of aerobic training is associated with a reduction in the calcineurin/NFAT signalling pathway in heart failure mice. J. Physiol. 587(Pt 15), 3899-3910. doi: 10.1113/jphysiol.2009. 173948

Olszanecka, A., Dragan, A., Kawecka-Jaszcz, K., Fedak, D., and Czarnecka, D. (2017). Relationships of insulin-like growth factor-1, its binding proteins, and cardiometabolic risk in hypertensive perimenopausal women. Metabolism 69, 96-106. doi: 10.1016/j.metabol.2017.01.005

Ooi, J. Y., Bernardo, B. C., and McMullen, J. R. (2014). The therapeutic potential of miRNAs regulated in settings of physiological cardiac hypertrophy. Future Med. Chem. 6, 205-222. doi: 10.4155/fmc.13.196

Palstra, A. P., Tudorache, C., Rovira, M., Brittijn, S. A., Burgerhout, E., van den Thillart, G. E., et al. (2010). Establishing zebrafish as a novel exercise model: swimming economy, swimming-enhanced growth and muscle growth marker gene expression. PLoS One 5:e14483. doi: 10.1371/journal.pone.0014483

Pelliccia, A., Culasso, F., Di Paolo, F. M., and Maron, B. J. (1999). Physiologic left ventricular cavity dilatation in elite athletes. Ann. Intern. Med. 130, 23-31. doi: 10.7326/0003-4819-130-1-199901050-00005

Pelliccia, A., Maron, B. J., Spataro, A., Proschan, M. A., and Spirito, P. (1991). The upper limit of physiologic cardiac hypertrophy in highly trained elite athletes. N. Engl. J. Med. 324, 295-301. doi: 10.1056/NEJM1991013132 40504

R Core Team (2020). "R: A Language and Environment for Statistical Computing." Vienna: $R$ Foundation for Statistical Computing. Available online at: https:// www.R-project.org/
Ravenscroft, G., Miyatake, S., Lehtokari, V. L., Todd, E. J., Vornanen, P., Yau, K. S., et al. (2013). Mutations in KLHL40 are a frequent cause of severe autosomalrecessive nemaline myopathy. Am. J. Hum. Genet. 93, 6-18. doi: 10.1016/j.ajhg. 2013.05.004

Rehfeldt, C., Renne, U., Sawitzky, M., Binder, G., and Hoeflich, A. (2010). Increased fat mass, decreased myofiber size, and a shift to glycolytic muscle metabolism in adolescent male transgenic mice overexpressing IGFBP-2. Am. J. Physiol. Endocrinol. Metab. 299, E287-E298. doi: 10.1152/ajpendo.00492.2009

Roostalu, U., and Strahle, U. (2012). In vivo imaging of molecular interactions at damaged sarcolemma. Dev. Cell 22, 515-529. doi: 10.1016/j.devcel.2011.12.008

Saltiel, A. R., and Kahn, C. R. (2001). Insulin signalling and the regulation of glucose and lipid metabolism. Nature 414, 799-806. doi: 10.1038/414799a

Schaloske, R. H., and Dennis, E. A. (2006). The phospholipase A2 superfamily and its group numbering system. Biochim. Biophys. Acta 1761, 1246-1259. doi: 10.1016/j.bbalip.2006.07.011

Schultz, R. L., Swallow, J. G., Waters, R. P., Kuzman, J. A., Redetzke, R. A., Said, S., et al. (2007). Effects of excessive long-term exercise on cardiac function and myocyte remodeling in hypertensive heart failure rats. Hypertension 50, 410-416. doi: 10.1161/HYPERTENSIONAHA.106.086371

Semenza, G. L. (2014). Hypoxia-inducible factor 1 and cardiovascular disease. Annu. Rev. Physiol. 76, 39-56. doi: 10.1146/annurev-physiol-021113-170322

Sharples, A. P., Al-Shanti, N., Hughes, D. C., Lewis, M. P., and Stewart, C. E. (2013). The role of insulin-like-growth factor binding protein 2 (IGFBP2) and phosphatase and tensin homologue (PTEN) in the regulation of myoblast differentiation and hypertrophy. Growth Horm. IGF Res. 23, 53-61. doi: 10. 1016/j.ghir.2013.03.004

Shimizu, H., Langenbacher, A. D., Huang, J., Wang, K., Otto, G., Geisler, R., et al. (2017). The Calcineurin-FoxO-MuRF1 signaling pathway regulates myofibril integrity in cardiomyocytes. eLife 6:e27955. doi: 10.7554/eLife.27955

Shimizu, I., Minamino, T., Toko, H., Okada, S., Ikeda, H., Yasuda, N., et al. (2010). Excessive cardiac insulin signaling exacerbates systolic dysfunction induced by pressure overload in rodents. J. Clin. Invest. 120, 1506-1514. doi: 10.1172/ JCI40096

Shioi, T., McMullen, J. R., Kang, P. M., Douglas, P. S., Obata, T., Franke, T. F., et al. (2002). Akt/protein kinase B promotes organ growth in transgenic mice. Mol. Cell Biol. 22, 2799-2809. doi: 10.1128/mcb.22.8.2799-2809.2002

Siscovick, D. S., Weiss, N. S., Fletcher, R. H., and Lasky, T. (1984). The incidence of primary cardiac arrest during vigorous exercise. N. Engl. J. Med. 311, 874-877. doi: 10.1056/NEJM198410043111402

Sousa Fialho, M. D. L., Abd Jamil, A. H., Stannard, G. A., and Heather, L. C. (2019). Hypoxia-inducible factor 1 signalling, metabolism and its therapeutic potential in cardiovascular disease. Biochim. Biophys. Acta Mol. Basis Dis. 1865, 831-843. doi: 10.1016/j.bbadis.2018.09.024

Strohecker, A. M., Joshi, S., Possemato, R., Abraham, R. T., Sabatini, D. M., and White, E. (2015). Identification of 6-phosphofructo-2-kinase/fructose2,6-bisphosphatase as a novel autophagy regulator by high content shRNA screening. Oncogene 34, 5662-5676. doi: 10.1038/onc.2015.23

Witteles, R. M., and Fowler, M. B. (2008). Insulin-resistant cardiomyopathy clinical evidence, mechanisms, and treatment options. J. Am. Coll Cardiol. 51, 93-102. doi: 10.1016/j.jacc.2007.10.021

Zhang, P., Yao, Q., Lu, L., Li, Y., Chen, P. J., and Duan, C. (2014). Hypoxiainducible factor 3 is an oxygen-dependent transcription activator and regulates a distinct transcriptional response to hypoxia. Cell Rep. 6, 1110-1121. doi: 10.1016/j.celrep.2014.02.011

Zhang, R., Yang, J., Zhu, J., and Xu, X. (2009). Depletion of zebrafish Tcap leads to muscular dystrophy via disrupting sarcomere-membrane interaction, not sarcomere assembly. Hum. Mol. Genet. 18, 4130-4140. doi: 10.1093/hmg/ ddp 362

Conflict of Interest: The authors declare that the research was conducted in the absence of any commercial or financial relationships that could be construed as a potential conflict of interest.

Copyright $\odot 2020$ Zhou, Zheng, Tang, Chen, Zhu, Peng, Wu and Zhu. This is an open-access article distributed under the terms of the Creative Commons Attribution License (CC BY). The use, distribution or reproduction in other forums is permitted, provided the original author(s) and the copyright owner(s) are credited and that the original publication in this journal is cited, in accordance with accepted academic practice. No use, distribution or reproduction is permitted which does not comply with these terms. 